\title{
Efeito da calcinação do resíduo de bauxita nas características reológicas e no estado endurecido de suspensões com cimento Portland
}

\author{
Effect of bauxite residue calcination on rheological and \\ hardened properties of suspensions with Portland cement
}

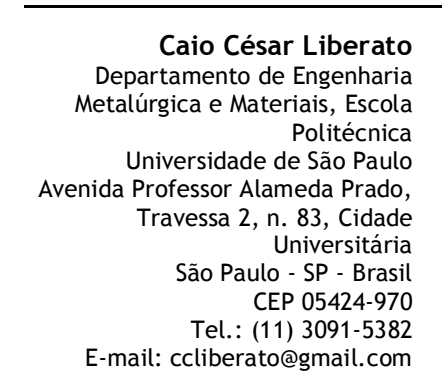

Roberto Cesar de Oliveira

Departamento de Engenharia de Construção Civil, Escola Politécnica Universidade de São Paulo Tel.: (11) 3091-5382

E-mail: rcorjau@gmail.com

Marcelo Montini Área de Aplicações, Desenvolvimentos

Alcoa Aluminio S.A. Rodovia Poços-Andradas, Km 10

Poços de Caldas - MG - Brasil CEP 37701-970 Tel.: (35) 3729-5541

marcelo.montini@alcoa.com.br

Jorge Borges Gallo Area de Aplicações, Desenvolvimentos

Alcoa Aluminio S.A. Rodovia Poços-Andradas, Km 10

Poços de Caldas - MG - Brasil CEP 37701-970

Tel.: (35) 3729-5541 Ramal 5541 E-mail: jorge.b.gallo@alcoa.com.br

Douglas Gouvea Departamento de Engenharia Metalúrgica e Materiais, Escola Politécnica Universidade de São Paulo Tel.: (11) 3091-5238

E-mail: dgouvea@usp.br

Rafael Giuliano Pileggi Departamento de Engenharia de Construção Civil, Escola Politécnica Universidade de São Paulo Tel.: (11) 3091-5382

E-mail: rafael.pileggi@poli.usp.br

\section{Caio César Liberato \\ Roberto Cesar de Oliveira Romano \\ Marcelo Montini \\ Jorge Borges Gallo \\ Douglas Gouvea \\ Rafael Giuliano Pileggi}

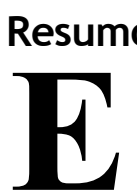

ste estudo ilustra o impacto da calcinação de resíduo de bauxita (RB) nas propriedades de suspensões formuladas com cimento Portland, tanto no estado fresco como no endurecido. As suspensões foram avaliadas contendo uma razão constante de água-cimento e teor de resíduo variando de $5 \%$ a $20 \%$ em peso e em substituição ao cimento. As propriedades reológicas e a resistência mecânica foram alteradas em função do aumento do teor de RB, mas a calcinação não teve influência no resultado final obtido, seja no estado fresco ou no endurecido. Assim, pode-se afirmar que a utilização de resíduo de bauxita, natural ou calcinada, em formulações com cimento Portland pode reduzir o consumo de cimento, sendo uma alternativa para a utilização de uma grande quantidade deste tipo de resíduo.

Palavras-chave: Resíduo de bauxita. Calcinação. Cimento. Reologia. Propriedades mecânicas.

\section{Abstract}

This research work shows the impact of bauxite residue (BR) calcination on the rheological and hardened properties of Portland cement suspensions. These suspensions were evaluated with water-to-cement ratio of 0.5 and variations of residue content from $5 \%$ to $20 \%$ in partial substitution of cement. Both properties had influence of BR content, but had a negligible influence of calcination.

Therefore, it can be inferred that the utilization of $B R$, in nature or calcinated, in formulations with Portland cement, may reduce the consumption of cement, being an alternative to use large amounts of this type of residue.

Keywords: Bauxite residue. Calcination. Cement. Rheology. Mechanical properties. 


\section{Introdução}

O desenvolvimento de aplicações para o resíduo de bauxita (RB) vem ganhando espaço nas pesquisas científicas e tecnológicas, em especial para o uso do rejeito em associação ao cimento (SINGH; UPADHAYAY; PRASAD, 1996, 1997; PERA; BOUMAZA; AMBROISE, 1997; ZHIHUA et al., 2003; TSAKIRIDIS; AGATZINI-LEONARDOU; OUSTADAKIS, 2004; SILVA FILHO; ALVES; DA MOTTA, 2007; ZHIHUA; YANNA; ZHONGZI, 2009; $\quad$ VANGELATOS; ANGELOPOULOS; BOUFOUNOS, 2009; LIBERATO et al., 2009; ZHANG et al., 2009; LIBERATO et al., 2011; ANTUNES; CONCEIÇÃO; NAVARRO, 2011; ÁLVAREZ et al., 2011; LOURENÇO et al., 2011; RIBEIRO et al., 2011; SENFF; HOTZA; LABRINCHA, 2011; TELESCA et al., 2011).

No entanto, nenhuma aplicação em grande escala foi desenvolvida, e o resíduo continua sendo acondicionado em reservatórios especialmente projetados para esse fim, apesar de reconhecidas desvantagens associadas ao processo (LIBERATO et al., 2011; ÁLVAREZ et al., 2011; RIBEIRO et al., 2011; SATERNUS, 2011; SENFF; HOTZA; LABRINCHA, 2011; TELESCA et al., 2011; KLAUBER; GRĂFE; POWER, 2011).

Duas características do resíduo podem dificultar a associação com materiais cimentícios: a elevada área de superfície específica; e o alto teor de sódio resultante da digestão do minério com $\mathrm{NaOH}$ (ZHANG et al., 2009; SENFF; HOTZA; LABRINCHA, 2011; RIBEIRO et al., 2011).

Uma área de superfície específica elevada reduz a distância de separação entre as partículas, aumenta a taxa de colisões e intensifica o movimento browniano, fazendo com que as forças de superfície (de curta distância) prevaleçam durante o fluxo (OLIVEIRA et al., 2000). Como consequência, aumenta a demanda de água para o amassamento do cimento, que, após o endurecimento, se torna mais poroso e permeável.

Tanto o aumento da porosidade como o da permeabilidade facilitam a difusão de materiais solúveis, que, no caso do sódio e de outros álcalis solúveis, podem provocar danos ambientais. Devese levar em consideração que o produto permanecerá sob a ação das intempéries. Por isso, é de extrema importância que não haja qualquer liberação de substâncias solúveis para o meio externo, além de possíveis degradações do produto.

Uma das alternativas estudadas para uma possível modificação das propriedades do RB e para tornálo mais apropriado à incorporação ao cimento sem uso demasiado de água foi o processo de calcinação. Além de permitir a redução da área de superfície específica e de reduzir a solubilidade de substâncias químicas indesejáveis, como sódio e flúor, possibilita alterar o tempo de pega, a expansibilidade e a reação álcali-agregado, devido a alterações na reatividade do rejeito (ZHANG et al., 2009; ZHANG; SUN; NIU, 2012; SENFF; HOTZA; LABRINCHA, 2011; RIBEIRO et al., 2011).

No entanto, não há um consenso geral sobre a eficácia do tratamento térmico na adequação do resíduo para aplicação em composições cimentícias. Apesar de alguns autores aprovarem a ideia, são reportados fatores negativos, principalmente no estado endurecido, que inviabiliza a utilização (SINGH; UPADHAYAY; PRASAD, 1996; TSAKIRIDIS; AGATZINILEONARDOU; OUSTADAKIS, 2004; SILVA FILHO; ALVES; DA MOTTA, 2007; SUSHIL; BATRA, 2008; ZHANG et al., 2009; ZHANG; SUN; NIU, 2012; RIBEIRO et al., 2011; ANTUNES; CONCEIÇÃO; NAVARRO, 2011; SENFF; HOTZA; LABRINCHA, 2011; GHOSH et al., 2011). Ademais, poucos trabalhos são encontrados na literatura com foco na caracterização reológica (LIBERATO et al., 2009; RIBEIRO et al., 2011; SENFF; HOTZA; LABRINCHA, 2011).

Assim, este estudo foi realizado com o objetivo de investigar o efeito da utilização de RB em formulações com cimento Portland e o impacto do processo de calcinação do rejeito nas propriedades reológicas e na resistência mecânica.

\section{Materiais e métodos de ensaio}

Cimento Portland contendo $20 \%$ de filler calcário e resíduo de bauxita do processo Bayer da planta de obtenção de alumina da Alcoa América Latina foram usados neste trabalho. Duas formas do resíduo foram aplicadas nas formulações: a primeira, in natura, seca a $105^{\circ} \mathrm{C}$, destorroada e peneirada; na segunda, o resíduo in natura foi calcinado a 800 oC por 1 hora, destorroado e peneirado.

Os ensaios foram realizados conforme apresentado a seguir:

(a) distribuição granulométrica a laser: foi medida em um equipamento Malvern, modelo Mastersizer $\mathrm{S}$ long bed Ver 2.19, com faixa de detecção de $0,05 \mu \mathrm{m}$ a $555 \mu \mathrm{m}$;

(b) medida de Área de Superfície Específica (ASE): a medida, baseada na adsorção física e

54 Liberato, C. C.; Romano, R. C. de O.; Montini, M.; Gallo, J. B.; Gouvea, D.; Pileggi, R. G. 
dessorção de gás na superfície da amostra sólida, foi realizada a partir do método de BET

(Braunauer, Emmet e Teller), em um equipamento Gemini 2375 - Micromeritics com pré-tratamento das amostras em temperatura de $200^{\circ} \mathrm{C}$ e pressão de $100 \mathrm{mmHg}$;

(c) picnometria de gás He: a densidade real dos pós foi determinada por picnometria de adsorção de gás He em um equipamento Multipicnometer, da marca Quantachrome MVP 5DC;

(d) Reometria Rotacional: os ensaios foram realizados em um reômetro AR550, TA

Instruments, usando geometria de placas paralelas. A taxa de cisalhamento foi alterada de $0 \mathrm{a}_{400 \mathrm{~s}^{-1} \mathrm{e}}$ depois retornou a 0 em $2 \mathrm{~min}$;

(e) Potencial Zeta: a mobilidade eletroforética em função do $\mathrm{pH}$ foi obtida em um equipamento Matec, modelo ESA 9800, Zeta Potential Analyzer. Uma suspensão com 5\% de RB foi preparada e atingiu $\mathrm{pH} 12,5$. A titulação foi realizada com ácido nítrico $1 \mathrm{M}$ até $\mathrm{pH} 2$;

(f) Resistência Mecânica: a tração na compressão diametral foi medida usando uma máquina de ensaio universal Instron, modelo 5569. As amostras, com diâmetro de $50 \mathrm{~mm}$ e espessura de $10 \mathrm{~mm}$, foram curadas por 7 dias em $25^{\circ} \mathrm{C}$ e $98 \%$ de umidade relativa; e

(g) procedimento de mistura: suspensões com proporção água/cimento de 2:1 em massa foram misturadas usando um equipamento Labortechnik RW20, IKA. A velocidade de rotação foi mantida constante em 1.200 RPM nos primeiros $2 \mathrm{~min}$ e, em seguida, aumentada para 2.500 RPM, tendo permanecido assim por mais $2 \mathrm{~min}$, na tentativa de promover melhor dispersão das partículas.

\section{Resultados e discussão}

A distribuição granulométrica, a área de superfície específica (ASE) e a densidade das matériasprimas são apresentadas na Figura 1 e na Tabela 1 .

Mesmo com similaridade na densidade real das matérias-primas, o resíduo de bauxita apresentou área específica muito maior que o cimento, independentemente do tratamento térmico. Entretanto, a calcinação em $800{ }^{\circ} \mathrm{C}$ foi responsável pela redução da área específica de $29,6 \mathrm{~m}^{2} / \mathrm{g}$ no resíduo in natura para $16,0 \mathrm{~m}^{2} / \mathrm{g}$.

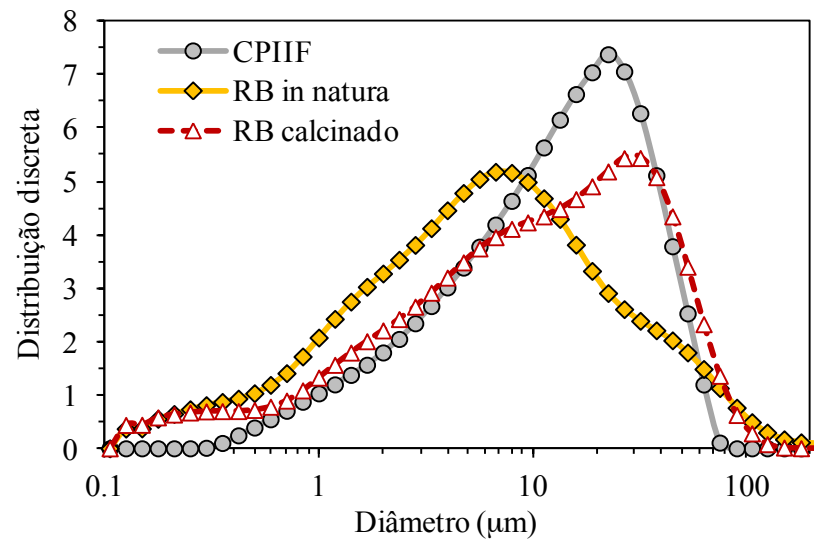

Figura 1 - Distribuição granulométrica do cimento e dos resíduos, seco ou calcinado

Tabela 1 - Propriedades físicas das matérias-primas

\begin{tabular}{l|c|c|c}
\hline \multicolumn{1}{c|}{ Propriedade } & RB & RB calcinado & CPIIF \\
\hline Área de superfície específica $\left(\mathrm{m}^{2} / \mathrm{g}\right)$ & 29,6 & 16,0 & 1,75 \\
Densidade real $\left(\mathrm{g} / \mathrm{cm}^{3}\right)$ & $2,99 \pm 0,04$ & $2,98 \pm 0,05$ & $3,0 \pm 0,04$ \\
$\mathrm{~d}_{10}(\mu \mathrm{m})$ & 0,6 & 0,9 & 2,3 \\
$\mathrm{~d}_{50}(\mu \mathrm{m})$ & 3,6 & 10,1 & 12,6 \\
$\mathrm{~d}_{90}(\mu \mathrm{m})$ & 19,0 & 41,4 & 35,7 \\
\hline
\end{tabular}




\section{Características das composições}

A partir da distribuição granulométrica e das proporções das matérias-primas na formulação, foram estimadas as composições granulométricas e a evolução da distribuição de tamanhos de partículas, conforme ilustrado na Figura 2. ̀̀ esquerda estão os resultados para as formulações com o RB in natura, e a direita, com o RB calcinado em $800 \quad{ }^{\circ} \mathrm{C}$. $\mathrm{Na}$ Tabela 2 são apresentados os valores de ASE resultantes nas composições e a distância de separação entre as partículas na pasta em função da variação das proporções entre cimento e RB.

A evolução da distribuição de partículas foi estimada utilizando-se como referência a composição sem RB, ou seja, subtraindo a porcentagem de partículas da composição de interesse $(\mathrm{x}=5 \%, 10 \%$ ou $20 \%$ de $\mathrm{RB}) \mathrm{da}$ porcentagem de partículas da formulação sem resíduo (\%xRB - \%0RB). Por isso, a curva da formulação-base (0RB) foi igual a 0 .
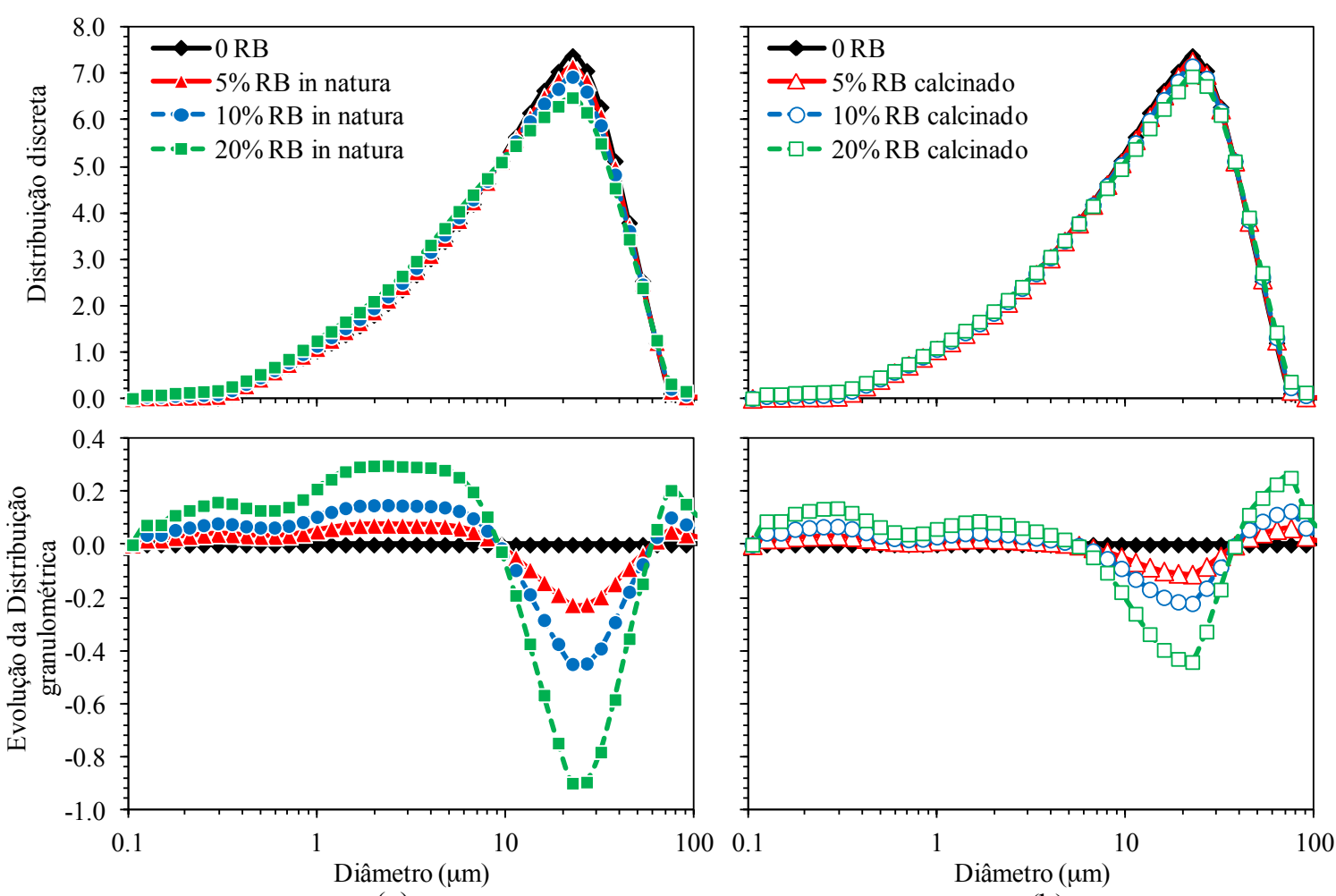

(a)

(b)

Figura 2 - Distribuição granulométrica das formulações com RB (a) in natura ou (b) calcinado - abaixo são mostrados os gráficos da evolução da distribuição granulométrica em função da variação do teor de RB

Tabela 2 - Estimativas de porosidade de empacotamento, ASE e distância de separação entre as partículas das composições

\begin{tabular}{l|l|c|c|c}
\hline \multirow{2}{*}{} & Teor de RB & $\begin{array}{c}\text { Área de superfície } \\
\text { específica }\end{array}$ & $\begin{array}{c}\text { Distância de separação } \\
\text { entre as partículas }\end{array}$ \\
\cline { 3 - 5 } & (\%-peso) & $\mathbf{( \mathbf { m } ^ { 2 } / \mathbf { g } )}$ & $(\boldsymbol{\mu m})$ \\
\hline \multirow{3}{*}{ In natura } & CPIIF & 0 & 1,75 & 0,515 \\
& $5 \%$ RB & 5 & 3,15 & 0,288 \\
& $10 \%$ RB & 10 & 4,54 & 0,201 \\
& $20 \%$ RB & 20 & 7,33 & 0,126 \\
\hline \multirow{3}{*}{ Calcinada } & $5 \%$ RB calc. & 5 & 2,46 & 0,367 \\
& $10 \%$ RB calc. & 10 & 3,18 & 0,286 \\
& $20 \%$ RB calc. & 20 & 4,60 & 0,198 \\
\hline
\end{tabular}

56 Liberato, C. C.; Romano, R. C. de O.; Montini, M.; Gallo, J. B.; Gouvea, D.; Pileggi, R. G. 
Fica nítido que a utilização do $\mathrm{RB}$ in natura nas formulações teve maior impacto do que o RB calcinado, seja na composição granulométrica ou no aumento da área específica global, visto que o resíduo calcinado apresenta tamanhos de partículas semelhantes ao cimento e ASE com quase metade do valor obtido para o resíduo in natura.

A quantidade de partículas com diâmetros no intervalo de 10-80 $\mu \mathrm{m}$ diminui com o aumento da quantidade de RB, ao mesmo tempo em que a porcentagem de partículas menores que $10 \mu \mathrm{m}$ aumenta. Esse efeito é intensificado nas composições com o resíduo in natura. Com o aumento da quantidade de partículas mais finas, há intensificação das forças de superfície no sistema como um todo e, para manter a consistência das suspensões, é prática comum a utilização de maior quantidade de água para o amassamento. Dessa forma, a distância de separação entre as partículas na pasta é menor quanto maior o teor de resíduo, o que dificulta o fluxo.

Devido a esse fato, em alguns trabalhos a aplicação do resíduo de bauxita em composições cimentícias é avaliada com diferentes consumos de água; por consequência, a conclusão é a de que a presença do RB deteriora as propriedades no estado endurecido (LIBERATO et al., 2009; RIBEIRO et al., 2011; SENFF; HOTZA; LABRINCHA, 2011).

Neste trabalho a quantidade de água foi mantida constante, as alterações na consistência foram avaliadas a partir de ensaios reológicos de fluxo contínuo e o efeito real da utilização de RB na resistência mecânica foi quantificado a partir de ensaios de compressão diametral, conforme apresentado nas próximas seções.

\section{Propriedades reológicas}

$\mathrm{Na}$ Figura 3 são apresentados os resultados dos ensaios reológicos nas suspensões com variações dos teores de RB. A tensão de cisalhamento foi quantificada em função da variação da taxa de cisalhamento. Em (a) estão os resultados das composições com RB in natura, e em (b), calcinado a $800^{\circ} \mathrm{C}$.

O comportamento reológico de fluidos pseudoplásticos com tensão de escoamento foi observado em todas as pastas cimentícias. A viscosidade, a tensão de cisalhamento, a área de histerese e os níveis de tixotropia positiva foram distintos, diretamente proporcionais ao aumento do teor de resíduo e independentes do processo de calcinação.

A calcinação não parece causar impacto relevante nas características reológicas das suspensões, diferentemente do que era esperado no início do trabalho. A principal proposta para utilização do tratamento térmico foi reduzir a ASE do resíduo com o intuito de diminuir a demanda de água para o amassamento.

Apesar de as suspensões com RB calcinado apresentarem menores ASE, a tensão de escoamento não sofreu alterações consideráveis, como pode ser visto na Figura 4. Na realidade, o resultado inverso foi observado, e nas composições com maior área específica os valores de tensão de escoamento foram sensivelmente inferiores.

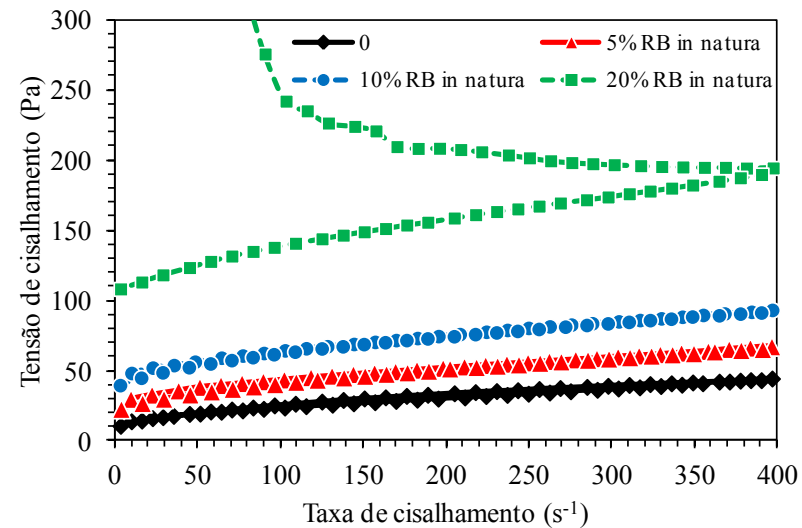

(a)

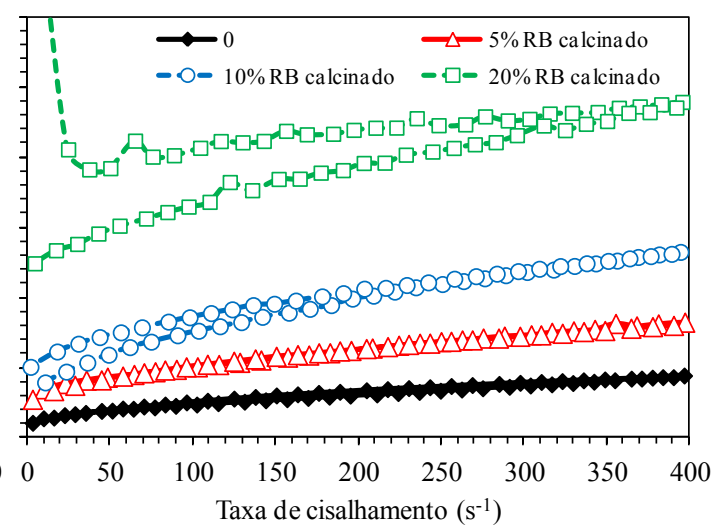

(b)

Figura 3 - Efeito do teor de RB e do tratamento térmico na tensão de cisalhamento das pastas cimentícias 


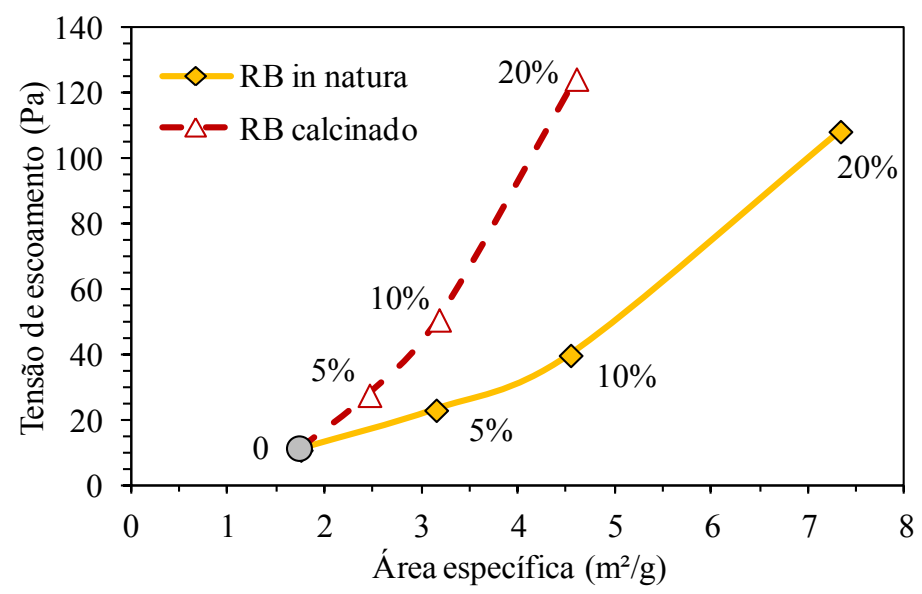

Figura 4 - Relação entre a tensão de escoamento e a área de superfície específica das composições

Uma explicação para o efeito observado é que, além de reduzir a ASE global, as partículas de RB apresentam carga superficial negativa para $\mathrm{pH}=$ 12 ( $\mathrm{pH}$ próximo ao da suspensão de cimento), conforme ilustrado na Figura 5. O potencial zeta do $\mathrm{RB}$ in natura foi de $-72 \mathrm{mV}$, enquanto para o resíduo calcinado foi de $-105 \mathrm{mV}$. No caso do cimento, o potencial de superfície foi de $+72 \mathrm{mV}$. Em contato com água, as partículas minerais adquirem cargas superficiais em consequência da ionização, adsorção e/ou dissolução iônicas, as quais podem influenciar no arranjo dos íons na vizinhança: íons com sinal oposto serão atraídos, e íons de mesma carga serão repelidos.

$\mathrm{O} \mathrm{pH}$ no ponto isoelétrico do resíduo in natura foi de 5,4 , e o observado para o resíduo calcinado foi de 7,2, ilustrando que existem consideráveis diferenças superficiais entre as duas matériasprimas. Em $\mathrm{pH}$ ácido o potencial zeta do resíduo de bauxita é positivo, e em regiões alcalinas é negativo, independentemente do tratamento térmico.

Como a carga superficial resultante no cimento é predominantemente positiva no início da reação de hidratação, devido à solubilização/absorção de íons $\mathrm{Ca} 2+$ na superfície das partículas, a interação cimento/resíduo apresenta tendência natural de aglomeração, independentemente do processo de calcinação (LYRA, 2010), afetando a tensão de escoamento dos materiais. No entanto, essa tendência foi mais acentuada nas composições com o RB calcinado do que com o RB in natura, devido ao maior potencial de superfície (negativo) observado, perturbando de forma mais crítica o fluxo.

Assim, mesmo com a redução da ASE do resíduo, os perfis de fluxo das suspensões foram semelhantes e independentes do tratamento térmico:

(a) nas formulações com o material in natura, a elevada área de superfície específica teve maior impacto nas características reológicas, devido à necessidade de maior quantidade de água para o recobrimento das partículas; e

(b) nas formulações com o resíduo calcinado em $800{ }^{\circ} \mathrm{C}$, o potencial de superfície governou as alterações nas propriedades reológicas, visto que induziu à aglomeração das partículas.

\section{Resistência mecânica}

Alguns trabalhos reportados em literatura atribuem a deterioração das propriedades mecânicas de formulações de cimento/RB à utilização do resíduo, mas na maioria dos casos o teor de água de amassamento é alterado para a manutenção da trabalhabilidade. Em outros trabalhos é reportado que as propriedades mecânicas são melhoradas devido à adição do RB (LIBERATO et al., 2009; RIBEIRO et al., 2011; SENFF; HOTZA; LABRINCHA, 2011).

No segundo caso, na grande maioria das vezes o consumo de água é mantido constante, sendo possível avaliar puramente o efeito da utilização do RB nas formulações, visto que esta é a única variável no ensaio.

Assim, neste trabalho foi seguida a mesma ideia, e os resultados dos testes mecânicos em função do teor de resíduo na composição são apresentados na Figura 6. Em (a) estão os resultados para as formulações com o RB in natura, e em (b), com o resíduo calcinado. 


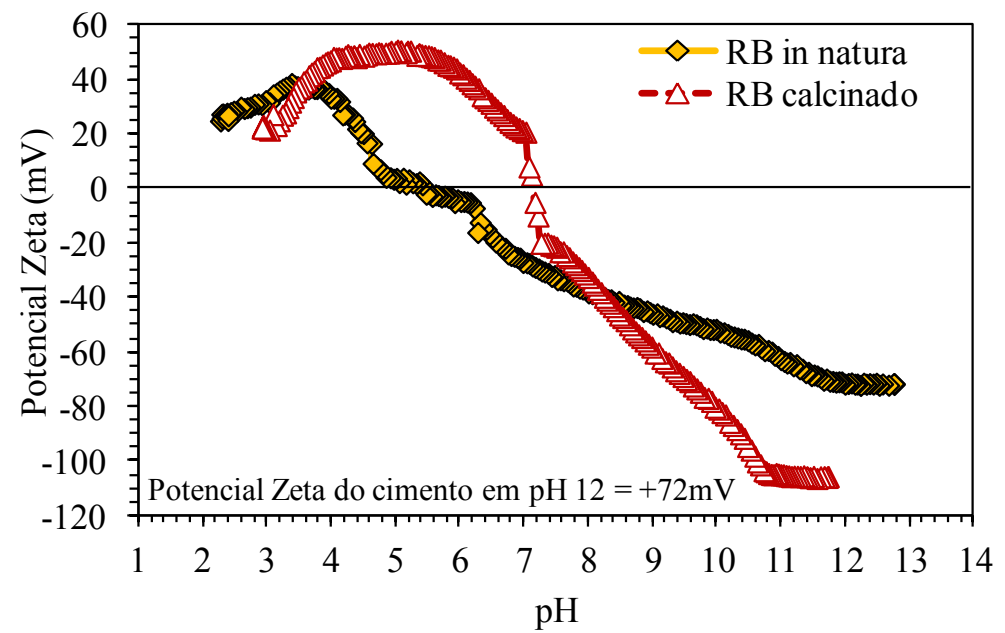

Figura 5 - Potencial zeta do resíduo de bauxita em função do pH

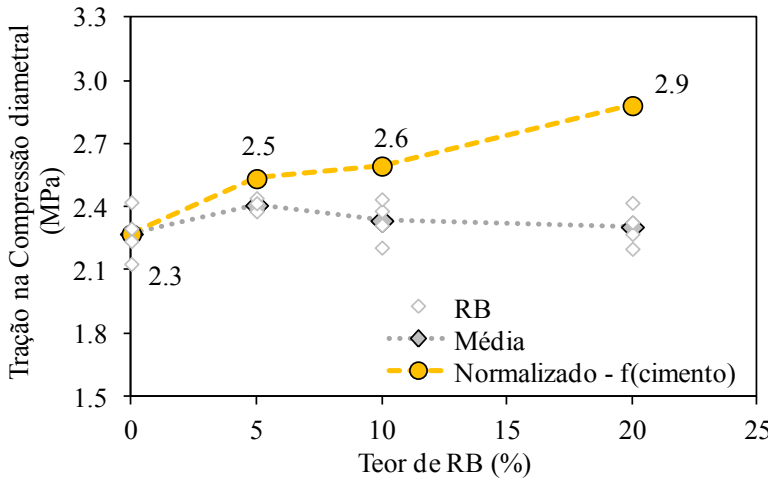

(a) Resultados das composições com RB in natura

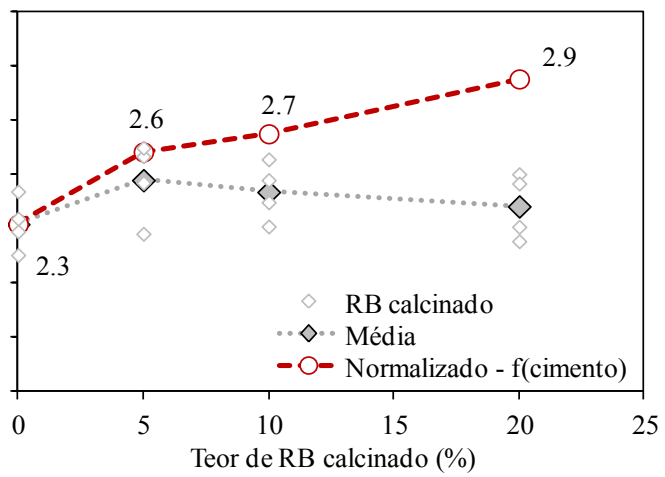

(b) RB calcinado

Figura 6 - Resistência à tração na compressão diametral

Duas formas de apresentação dos resultados foram utilizadas:

(a) os losangos sem preenchimento representam os resultados puros de tração na compressão diametral (quatro pontos para cada amostra), e os losangos com preenchimento são as médias obtidas; e

(b) os círculos representam a resistência à tração normalizada em função da quantidade de cimento na composição $(100 \%, 95 \%, 90 \%$ ou $80 \%)$. Esta segunda forma de avaliação foi adotada porque, com o aumento do teor de resíduo de bauxita, a quantidade de cimento foi reduzida.

A utilização do RB potencializou a eficiência do ligante, sendo as alterações na resistência mecânica proporcionais ao aumento do teor e independentes do tratamento térmico do resíduo.

Mesmo com quantidades menores de cimento na formulação, os valores médios de resistência mecânica foram superiores ao valor atingido na amostra de referência, ou seja, 2,3 $\mathrm{MPa}$. Porém, comparando-se somente as amostras com RB, foi observada uma sensível redução na tensão de ruptura com o aumento do teor de $5 \%$ para $20 \%$.

No entanto, essa forma de avaliação não ilustra realmente o potencial da utilização do resíduo de bauxita nas composições cimentícias, visto que as formulações apresentam diferentes teores de ligante, matéria-prima responsável pelo desenvolvimento da resistência mecânica.

Normalizando-se os resultados em função da quantidade de cimento, fica clara a diferença de resistência mecânica das formulações, demonstrando um aumento de resistência mesmo com a redução do consumo de cimento. Em outras palavras, se o consumo de cimento fosse mantido constante, a resistência mecânica dos materiais com resíduo de bauxita seria maior do que o material de referência. Nos casos avaliados foi observado aumento de até $25 \%$ na tensão de ruptura, dependendo do teor de $\mathrm{RB} e$ independentemente do processo de calcinação.

Deve ser salientado que o processo de calcinação do RB foi indiferente nas alterações reológicas ou 
no estado endurecido, mas pode ser uma alternativa interessante no caso de aplicações do rejeito em composições cimentícias. Estas estarão sujeitas às intempéries naturais, o que pode reduzir a quantidade de materiais perigosos liberados ao meio ambiente. No entanto, essas avaliações ainda serão realizadas e apresentadas em trabalhos futuros.

Ainda não está muito claro se o sódio ou outros metais são solubilizados ou facilitam a reação álcali-agregado após o endurecimento do material cimentício, dificultando a difusão dessa prática no setor de construção civil.

Uma possível alternativa para reduzir a lixiviação de álcalis é a redução da porosidade e dos canais permeáveis na estrutura do material endurecido. Como a quantidade de água para o amassamento geralmente é alta, devido às características do resíduo, a microestrutura gerada ainda não é a ideal. Por outro lado, na tentativa de reduzir a reação álcali-agregado, a redução da quantidade de cimento é importante.

Uma alternativa que tem sido estudada, a qual deverá ser apresentada em trabalhos futuros, é a aplicação dos conceitos de estabilização de partículas através da utilização de aditivos dispersantes. A ideia é obter suspensões com baixa viscosidade utilizando a menor quantidade de água possível, visando tornar a microestrutura menos porosa e permeável.

\section{Conclusões}

A substituição parcial do cimento por resíduo de bauxita, seja in natura ou calcinado, afetou a área específica global das formulações, resultando em alterações nas características reológicas das suspensões.

A calcinação reduziu a área específica do $R B$, mas não foi suficiente para facilitar o fluxo das suspensões, pois, paralelamente, houve alteração no potencial de superfície do rejeito. $\mathrm{Na}$ somatória dos efeitos, as propriedades reológicas obtidas nas suspensões com o RB calcinado foram similares às observadas para as suspensões com o RB in natura. Portanto, o tratamento térmico não alterou as propriedades dos materiais durante o fluxo.

Com a utilização do RB nas formulações, mantendo-se a quantidade de água constante, foi possível obter produtos com aumento da resistência mecânica aliado à redução do consumo de cimento.

O processo de calcinação não afetou a tensão de ruptura, sendo observados resultados similares aos das suspensões com o resíduo in natura.
Pode-se afirmar que a utilização de RB, seja in natura ou calcinado, em composições cimentícias não deteriora a resistência mecânica e, na realidade, é uma forma de aplicação de um rejeito industrial em grande quantidade que permite a redução do consumo de cimento sem perda de rigidez do produto. Essa prática pode ser uma alternativa interessante para o setor de construção civil, mas ainda depende de estudos futuros, pois a elevada quantidade de substâncias perigosas na composição pode ser um problema ambiental, no caso de lixiviação ou solubilização.

\section{Referências}

ÁLVAREZ, L. et al. Microstructure and Durability of Cements Containing Red Mud. In: INTERNATIONAL CONGRESS ON THE CHEMISTRY OF CEMENT, 13., Madrid, 2011. Proceedings... Madrid, 2011.

ANTUNES, M. L. P.; CONCEIÇÃO, F. T.; NAVARRO, G. R. B. Caracterização da Lama Vermelha Brasileira (Resíduo do Refino da Bauxita) e Avaliação de Suas Propriedades Para Futuras Aplicações. In: INTERNATIONAL WORKSHOP ADVANCES IN CLEANER PRODUCTION, 3., São Paulo, 2011.

Proceedings... São Paulo, 2011.

GHOSH, I. et al. Leaching of Metals From Fresh and Sintered Red Mud. Journal of Hazardous Materials, v. 185, n. 2/3, p. 662-668, jan. 2011.

KLAUBER, C.; GRĂFE, M.; POWER, G. Bauxite Residue Issues: II. options for residue utilization. Hydrometallurgy, v. 108, n. 1/2, p. 11-32, jun. 2011.

LIBERATO, C. C. et al. Impact of Bauxite Residue in Cement Pastes on the Hardened State Properties. In: ARESTY UNDERGRADUATE RESEARCH SYMPOSIUM, New Jersey, 2011. Proceedings... New Jersey, 2011

LIBERATO, C. C. et al. Impacto das Características Fsicas de Lama Vermelha nas Propriedades de Pastas Cimentícias. In: SIMPÓSIO INTERNACIONAL DE INICIAÇÃO CIENTÍFICA DA UNIVERSIDADE DE SÃO PAULO, 17., São Carlos, 2009. Anais... São Paulo: USP, 2009.

LYRA, J. S. Estudo da Influência de Policarboxilato Comercial na Hidratação, Reologia e Físico-Química de Superfície do Cimento. 99 f. São Paulo, 2010. Dissertação (Mestrado em Engenharia Metalúrgica e de Materias) - Escola Politécnica, Universidade de São Paulo, São Paulo, 2010. 
LOURENÇO, R. R. et al. Use of Bauxite Residue as a Source of $\mathrm{Al} 2 \mathrm{O} 3$ and $\mathrm{Fe} 2 \mathrm{O} 3$ in the Preparation of Portland Cement Clinker. In: International Congress on the Chemistry of Cement, 13., Madrid, 2011. Proceedings... Madrid, 2011.

OLIVEIRA, I. R. et al. Dispersão e

Empacotamento de Partículas: princípios e aplicações em processamento cerâmico. São Paulo: Fazendo Arte Editorial, 2000. 224 p.

PERA, J.; BOUMAZA, R.; AMBROISE, J. Development of a Pozzolanic Pigment From Red Mud. Cement and Concrete Research, v. 27, n. 10, p. 1513-1522, 1997.

RIBEIRO, D. V. et al. Estudo de Eventuais Patologias Associadas ao Uso da Lama Vermelha em Argamassas Colantes e de Revestimento. Cerâmica Industrial, v. 16, n. 1, p. 31-42, jan./fev. 2011.

SATERNUS, M. Bayern's Method of A12O3 Production: possibilities of red mud disposal and utilization. Solid State Phenomena, v. 176, p. 1112, 2011 .

SENFF, L.; HOTZA, D.; LABRINCHA, J. A. Effect of Red Mud Addition on the Rheological Behavior and on Hardened State Characteristics of Cement Mortars. Construction and Building Materials, v. 25, n. 1, p. 163-170, 2011.

SILVA FILHO, E. B.; ALVES, M. C. M.; DA MOTTA, M. Lama Vermelha da Indústria de Beneficiamento de Alumina: produção, características, disposição e aplicações alternativas. Revista Matéria, v. 12, n. 2, p. 322338, 2007.

SINGH, M., UPADHAYAY, S. N., PRASAD, P. M. Preparation of Special Cements From Red Mud. Waste Management, v. 16, n. 8, p. 665-670, 1996.

SINGH, M.; UPADHAYAY, S. N.; PRASAD, P. M. Preparation of Iron Rich Cements Using Red Mud. Cement and Concrete Research, 27, n. 7, p. 1037-1046, 1997.

SUSHIL, S., BATRA, V. S. Catalytic Applications of Red Mud, an Aluminium Industry Waste: a review. Applied Catalysis B: Environmental, v. 81, n. $1 / 2$, p. $64-77,2008$.
TELESCA, A. et al. Calcium Sulfoaluminate Cements Obtained From Bauxite-Free Raw Mixes. In: INTERNATIONAL CONGRESS ON THE CHEMISTRY OF CEMENT, 13., Madrid, 2011. Proceedings... Madrid, 2011.

TSAKIRIDIS, P. E.; AGATZINI-LEONARDOU, S.; OUSTADAKIS, P. Red Mud Addition in the Raw Meal For the Production of Portland Cement Clinker. Journal of Hazardous Materials, v. 116, n. 1/2, p. 103-110, 2004.

VANGELATOS, I.; ANGELOPOULOS, G. N.; BOUFOUNOS, D. Utilization of Ferroalumina as Raw Material in the Production of Ordinary Portland Cement. Journal of Hazardous Materials 168, n. 1, p. 473-478, 2009.

WESTMAN, A. E. R., HUGILL, H. R. The Packing of Particle. Journal of American Ceramic Society, v. 13, n. 10, p. 767-779, 1930.

ZHANG, N. et al. Early-Age Characteristics of Red Mud-Coal Gangue Cementitious Material. Journal of Hazardous Materials, v. 167, n. 1/3, p. 927-932, ago. 2009.

ZHANG, J.; SUN, H.; NIU, X. Mechanism of the Improved Cementitious Activity in Co-Calcined Red Mud. Advanced Material Research, v. 347353, p. 1106-1112, 2012.

ZHANG, N. et al. Evaluation of Blends BauxiteCalcination-Method Red Mud With Other Industrial Wastes as a Cementitious Material: properties and hydration characteristics. Journal of Hazardous Materials, v. 185, n. 1, p. 329-335, 2011.

ZHIHUA, P. et al. Properties and Microstructure of the Hardened Alkali-Activated Red Mud-Slag Cementitious Material. Cement and Concrete Research, v. 33, n. 9, p. 1437-1441, 2003.

ZHIHUA, P.; YANNA, Z.; ZHONGZI, X. Strength Development and Microstructure of Hardened Cement Paste Blended With Red Mud. Journal of Wuhan University Technology Material, v. 24, p. 161-165, 2009.

\section{Agradecimentos}

Os autores agradecem à Capes e à Alcoa América Latina pelo suporte na realização do trabalho.

\section{Revista Ambiente Construído}

Associação Nacional de Tecnologia do Ambiente Construído Av. Osvaldo Aranha, $99-3^{\circ}$ andar, Centro Porto Alegre - RS - Brasil CEP $90035-190$

Telefone: +55 (51) 3308-4084 Fax: +55 (51) 3308-4054

www.seer.ufrgs.br/ambienteconstruido

E-mail: ambienteconstruido@ufrgs.br 